\title{
Molecular motors in a tight spot
}

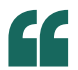

We discovered that by simply contracting or expanding the bowl size, depending on the percentage water, the rotary behaviour of the light-driven molecular motor could be controlled

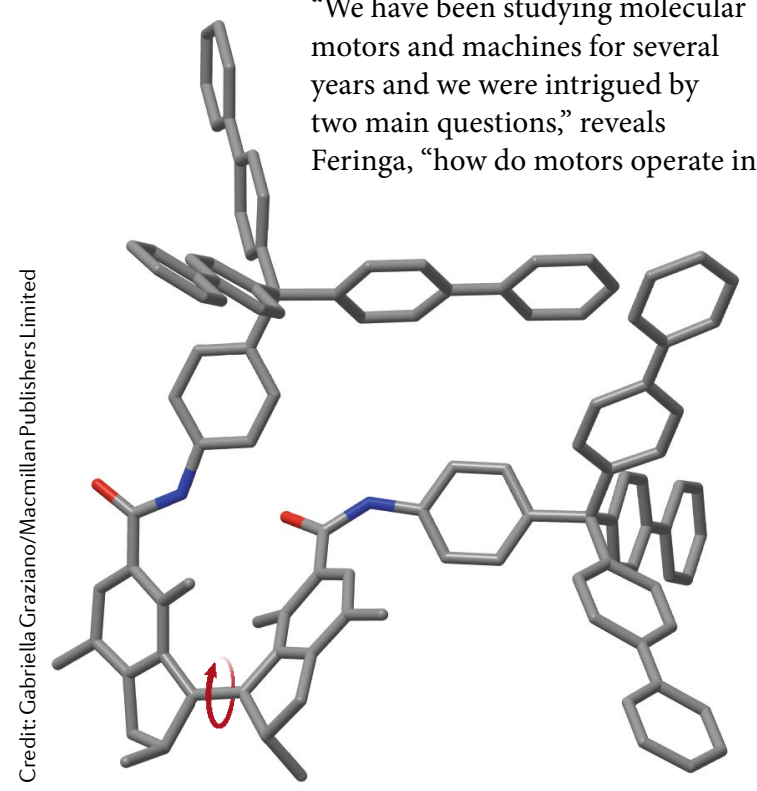

confined space? And how do pendant moieties of increasing size affect the rotary motion?" Furthermore, molecular motors have been studied so far mostly in organic solvents. Understanding the behaviour of these molecules in aqueous media is a very timely challenge that needs to be addressed if they are to be applied in biotechnological settings.

Feringa and co-workers have previously explored the self-assembly of water-soluble molecular motors in their design of a supramolecular muscle. In the present study, the researchers looked instead at the behaviour, in water, of a motor featuring hydrophobic moieties. The newly developed motor comprises an overcrowded alkene core and two rigid, highly hydrophobic tris-bisphenyl units. These motors are soluble in organic solvents, such as tetrahydrofuran, but the addition of water (non-solvent) initiates their aggregation into bowl-shaped particles. The aggregation mechanism in this case is different to that observed for large amphiphilic molecules, which assemble into spherical objects as a result of their molecular properties. The aggregation of these small hydrophobic molecules into bowl-shaped particles is driven, instead, by the ratio of solvent to non-solvent, independently of their molecular properties. In fact, the same mechanism has been observed for several other organic molecules, including polystyrene, polyvinyl chloride and Nile Red.

The dimensions of the bowlshaped particles can be controlled by tuning the solvent-non-solvent ratio. "We discovered that by simply contracting or expanding the bowl size, depending on the percentage water, the rotary behaviour of the light-driven molecular motor could be controlled," explains Feringa. In dilute solution, the investigated molecular motor performs a unidirectional $360^{\circ}$ rotation consisting of four steps: photoisomerization, thermal helix inversion, a further photoisomerization and a final thermal helix inversion that closes the cycle. This rotation is completely impeded in the solid state, but it can be controlled when the rotors are confined in small volumes. Depending on the size of the bowl-shaped particle, the thermal helix inversion of the motor can proceed forward or backward. "This is an amazing discovery, which shows that isomerization barriers and rotary motion in a molecular system can be controlled by solvent ratios and degree of aggregation. As far as we know, these findings are unprecedented," remarks Feringa.

The ability to control molecular aggregation and rotation by simply tuning the solvent-non-solvent ratio opens the path to a number of unexplored possibilities. "Can we use the motors as stirrers or mixers at the nanoscale? Can we perform reactions in these confined systems or use them to deliver cargo triggered by the molecular motion?" asks Feringa. "We could develop functional nanocontainers. How nice it can be!"

Gabriella Graziano

ORIGINAL ARTICLE Franken, L. et al. Solvent mixing to induce molecular motor aggregation into bowl-shaped particles: underlying mechanism, particle nature, and application to control motor behavior.J.Am. Chem. Soc., https://doi.org/10.1021/ jacs.8b03045 (2018). FURTHER READING Chen, J. et al. Artificial muscle-like function from hierarchical supramolecular assembly of photoresponsive molecular motors. Nat. Chem. 10, 132-138 (2018) 